\title{
FIR and sub-mm line observations of AGB and post-AGB nebulae
}

\author{
Valentín Bujarrabal ${ }^{1}$ \\ ${ }^{1}$ Observatorio Astronómico Nacional, Ap 112, E-28803 Alcalá de Henares, Spain \\ email: v.bujarrabal@oan.es
}

The existing information on asymptotic giant branch (AGB) and post-AGB objects from FIR and submm spectroscopy is still scarce. Observations from the ground are often very difficult to calibrate and show low $\mathrm{S} / \mathrm{N}$ ratios. However, we expect to obtain in the near future an impressive amount of high-quality data, from the Herschel space telescope. ALMA is also expected to provide high-quality maps of submm lines.

AGB stars eject significant amounts of gas, which forms spherical circumstellar envelopes (CSEs) expanding at moderate velocity (e.g. Habing 1996). AGB CSEs have been systematically observed by means of mm-wave molecular lines, for instance the intense low- $J$ rotational transitions of CO $(J=1-0$ and $J=2-1)$. Such lines are known to probe the bulk of the circumstellar material, requiring very low excitation conditions. However, they are not efficient in discriminating gas with relatively high excitation (over $\sim 100 \mathrm{~K}$ ). So, they are not very useful to study the inner CSE, at distances smaller than $\sim 10^{16} \mathrm{~cm}$. For such a purpose, we need observations of lines requiring intermediate excitation conditions, i.e. between about $100 \mathrm{~K}$ and $1000 \mathrm{~K}$, in almost all cases at submm and FIR wavelengths. The processes relevant to explain mass loss actually take place in these inner layers: pulsation of shells very close to the star and dust formation at a somewhat larger distance. The study of inner shells is crucial to understand those processes, and, therefore, AGB and post-AGB evolution, which is thought to be driven by the very copious mass ejection of these stars.

Preliminary works including submm lines (mostly of $\mathrm{CO}$ ) have been particularly useful for measuring the temperature profiles in AGB CSEs and for checking the presence of variations in the mass-loss rate in the last $1000 \mathrm{yr}$ (e.g. Groenewegen et al. 1998, Teyssier et al. 2006, Decin et al. 2007, Ramstedt et al. 2008). The temperature was confirmed to vary with distance to the star following approximately a potential law, $T \propto 1 / r^{\alpha}$, with $\alpha \sim 1$. In general, the data are compatible with constant mass-loss rate; in one object, IRC +10011 , a decrease by at least a factor 10 of the mass-loss rate took place about $800 \mathrm{yr}$ ago. We expect that data in the near future will very significantly increase our knowledge on this topic. Some existing space observations have also been useful to detect water vapor emission in O-rich and even C-rich stars (see Maercker et al. 2008, 2009; Melnick et al. 2001, González-Alfonso et al. 2007). However, SWAS and ODIN data only contain the very low-excitation and opaque $1_{1,0}-1_{1,1}$ line, and ISO detected only opaque lines using very low spectral resolution. Therefore, the interpretation of the existing data is very preliminary and in need of more observations to refine models.

As mentioned, the end of the AGB phase is thought to be due to the huge amounts of material (most of the initial stellar mass) ejected. The process is spectacular. In less than $\sim 1000 \mathrm{yr}$, the large and cold red giant, surrounded by a spherical, slowly expanding CSE, becomes a tiny and very hot blue dwarf, around which a young planetary nebula (PN) shows, in general, a clear axial symmetry and fast bipolar flows. The appearance of the central star is due to the ejection of all stellar layers over the core, which is now visible (the blue dwarf). The nebula evolution is thought to be due to shock interaction between the fossil AGB envelope and post-AGB axial jets carrying very high energy and momentum (e.g. Bujarrabal et al. 2001). The result is that huge amounts of material (a fraction of a solar mass) are accelerated to $50-100 \mathrm{kms}^{-1}$ in just a few hundred years. The physical processes at work, including both the wind interaction and the fast wind ejection, are still not well understood.

Young PNe have been well observed thanks to mm-wave molecular lines. Accurate maps of CO emission (e.g. Sánchez Contreras et al. 2004) reveal the density and velocity distribution of most of the nebular gas. In general, the detected gas is always in expansion; only for one object, the Red Rectangle, has a rotating disk been well mapped (Bujarrabal et al. 2005), and whose presence, if confirmed to be systematic in these objects, could explain the energetic post-AGB 
jets. Again, such observations are not efficient in discriminating warm regions, which must be studied to understand the PN shaping by shocks propagating in the massive component and the innermost rotating layers. Results from submm observations are still rare (e.g. Nakashima et al. 2007) and have not yielded conclusive results. We expect that future data will be crucial to improve our knowledge on the evolution of these objects.

Finally, I would like to mention interest in the study of PDRs in young PNe. PDRs appear in post-AGB evolution as an intermediate phase between the cold and molecule-rich AGB CSEs and the very hot gas in old PNe, which is fully ionized by the UV stellar field. PDRs are warm and rich in neutral or slightly-ionized plasma. Their study requires almost necessarily space telescopes. ISO detected some PDR tracers, namely fine-structure lines of O I, C II and some other atom (Castro-Carrizo et al. 2001, Fong et al. 2001). C II was particularly efficient to measure the total PDR mass, while the O I line at $63 \mu \mathrm{m}$ was useful to check models and study the excitation conditions. Massive (close to $1 \mathrm{~ms}^{-1}$ ) PDRs were found in some young PNe, like NGC 7027 and NGC 6302. But the lack of sensitivity and spectral resolution severely hampered these works, and systematic studies of this transitional component must still be performed.

\section{References}

Bujarrabal, V., Castro-Carrizo, A., Alcolea, J., \& Sánchez Contreras, C. 2001, A\&̈A 377, 868

Bujarrabal, V., Castro-Carrizo, A., Alcolea, J., \& Neri, R. 2005, A $\& A$ 441, 1031

Castro-Carrizo, A., Bujarrabal, V., Fong, D., et al. 2001, A\&\&A 367, 674

Decin, L., Hony, S., \& de Koter, A. 2007, A\&A 475, 233

Fong, D., Meixner, M., Castro-Carrizo, A., et al. 2001, A $\& A$ 367, 652

González-Alfonso, E., Neufeld, D.A., \& Melnick, G. J. 2007, ApJ 669, 412

Groenewegen, M. A. T.; van der Veen, W. E. C. J., \& Matthews, H. E. 1998, A\&A 338, 491

Habing, H. J. 1996, A\&AR 7, 97

Maercker, M., Schöier, F. L., Olofsson, H., et al. 2008, A\&A 479, 779

Maercker, M., Schöier, F. L., Olofsson, H., et al. 2009, A\&A 494, 243

Melnick, G. J., Neufeld, D. A., Ford, K. E. S., et al. 2001, Nature 412, 160

Nakashima, J., Fong, D., Hasegawa, T., et al. 2007, AJ 134, 2035

Ramstedt, S., Schöier, F. L., Olofsson, H., \& Lundgren, A. A. 2008, A\&\&A 487, 645

Sánchez Contreras, C., Bujarrabal, V., \& Castro-Carrizo, A. 2004, ApJ 617, 1142

Teyssier, D., Hernández, R., Bujarrabal, V., et al. 2006, A\&A 450, 167 